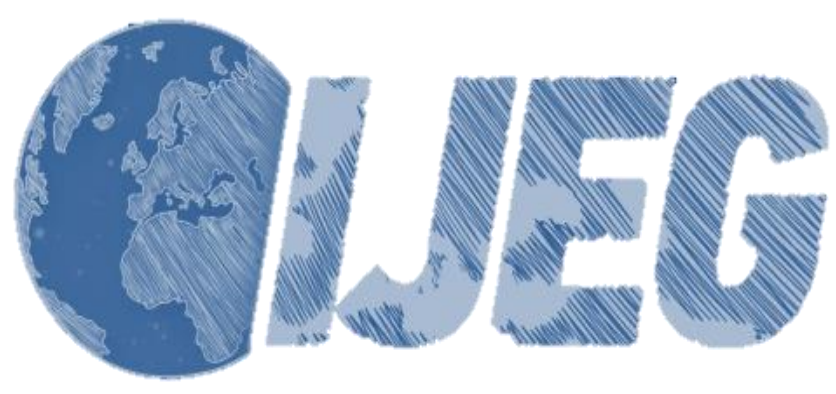

International Journal of Engineering and Geosciences (IJEG), Vol; 5, Issue; 3, pp. 144-149, October, 2020, ISSN 2548-0960, Turkey, DOI: 10.26833 ijeg. 648847

\title{
RADAR CROSS SECTION ANALYSIS OF UNMANNED AERIAL VEHICLES USING PREDICS
}

\author{
Caner Özdemir ${ }^{1,2 *}$ \\ ${ }^{1}$ Mersin University, Engineering Faculty, Department of Electrical-Electronics Engineering, Mersin, Turkey \\ (cozdemir@ mersin.edu.tr); ORCID 0000-0003-2615-4203 \\ ${ }^{2}$ Emtech Information Technologies Corporation, Mersin Technological Development Zone, Yenişehir, Mersin, \\ Turkey (caner. ozdemir@emtechbilisim.com)
}

*Corresponding Author, Received: 20/11/2019, Accepted: 29/01/2020

\begin{abstract}
In this study, a quantitative radar cross section (RCS) analysis of different unmanned aerial vehicle (UAV) models were accomplished by means of a series of RCS simulations. The simulations were carried out by high-frequency RCS simulation and analysis tool called PREDICS. To quantify the RCS features of the UAV model, both the anglevariation and frequency-variation simulations for all polarization excitations were performed. The results of the simulations suggested that RCS values were dramatically varying with respect to look angle with some special angles providing the large values of RCS. Generally, the RCS values of the UAV model was increasing with frequency as expected. A quantitative radar detection range analyses were also accomplished to assess the visibility of both the military-type and civil-type UAV models. The outcome of these studies has suggested that large-size UAV model can be easily detected by a high-sensitive radar on the ranges of tens of kilometers while these numbers reduce to a few kilometers for a civilian UAV model that is much smaller than the its military counterpart.
\end{abstract}

Keywords: Radar Cross Section, Unmanned Aerial Vehicle, Radar Visibility, RCS simulation 


\section{INTRODUCTION}

For the last decade, the number of Unmanned Aerial Vehicles (UAVs) that are being used in numerous applications has been extensively increased due to growing demand from both the military and civil sectors (Thite et al., 2012; Pieraccini et al., 2017; Ananenkov, et $a l ., 2018)$. One of the use of UAVs is for remote sensing applications (Ulvi et al., 2016; Akar, 2017, Ulvi, 2018). In a parallel manner, the need to detect such vehicles becomes really crucial for the safety on people. In military practices, UAVs are generally being used for reconnaissance, surveillance and targeted attacks (Ryapolov, et al., 2014). Therefore, it becomes very vital to sense and also make them ineffective before completing their offensive missions to avoid life losses and injuries. While their military usages provide direct danger for human health and life, their civil usages may sometimes provide risky and hazardous situations like accidents. For example, UAVs can be a serious threat for the flight safety according to Aviation authorities. It is no doubt that drones and small UAVs may not be perceived by the radar of either airport's tower and/or from airplanes that are coming in or going out from the runways. Furthermore, small, civilian-type UAVs can also be used offensive purposes such as terrorist attacks. Therefore, there is a need for assessing and analyzing the radar visibility characteristics of UAVs by calculating the radar cross section (RCS) of such vehicles.

In this paper, we represent a study for calculating and analyzing the RCS values of some generic UAVs at Xband of frequencies. The RCS simulations have been carried out by our recently developed RCS simulation and analysis software called PREDICS (Özdemir et al, 2014a; Özdemir et al, 2014b). The simulations are conducted in aiming to evaluate the detectability of UAVs by radar sensors, especially at X-band. For this purpose, a quantitative analysis and assessment on the detection of UAV-type targets by a nominal radar has been made.

For this paper, two different generic UAV models are taken into account. These models are quite similar to the ones that are commonly used in both military and civil applications.

\section{RCS SIMULATION OF A MILITARY- TYPE UAV MODEL: "MIL-UAV"}

The computer aided design (CAD) of UAV model named as Mil-UAV that was used in PREDICS simulation can be seen in Fig.1. This model was selected because it has very similar characteristics of military UAVs that are usually significantly bigger than civil ones. The perspective view of Mil-UAV is seen Fig.1(a). The top and front view of the model are shared in Fig. 1(b). As it can be read from the figure that the wing extend of Mil-UAV is $14.73 \mathrm{~m}$. The side view of Mil-UAV is plotted in Fig. 1(c). The total length and height of the model are $8.22 \mathrm{~m}$ and $2.40 \mathrm{~m}$, respectively. During the PREDICS simulation, the material of the model is assumed to be perfect electric conductor (PEC).

RCS simulation of the Mil-UAV has been accomplished by the PREDICS simulator (Özdemir et al, 2014a; Özdemir et al, 2014b). PREDICS is a fast and effective simulation tool for the fast and accurate calculation of RCS from electrically large and complex- shaped platforms at high frequencies. The detailed information about PREDICS tool; including the theory behind the code and other technical features can be reached at (Özdemir et al, 2014a; Özdemir et al, 2014b; Kirık et al, 2019). The main graphical user interface (GUI) screen of PREDICS for the simulation of Mil-UAV is viewed in Fig. 2.

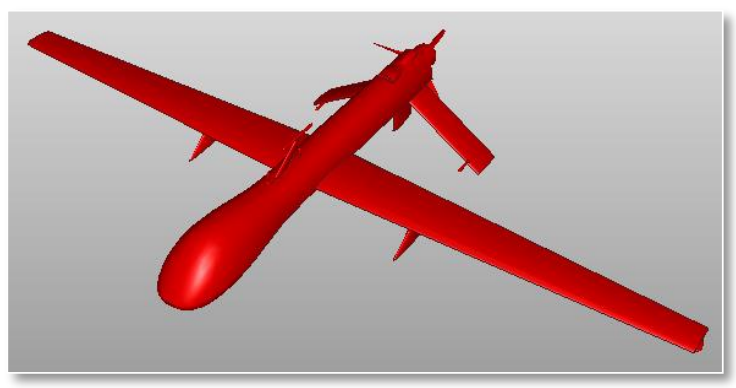

(a)

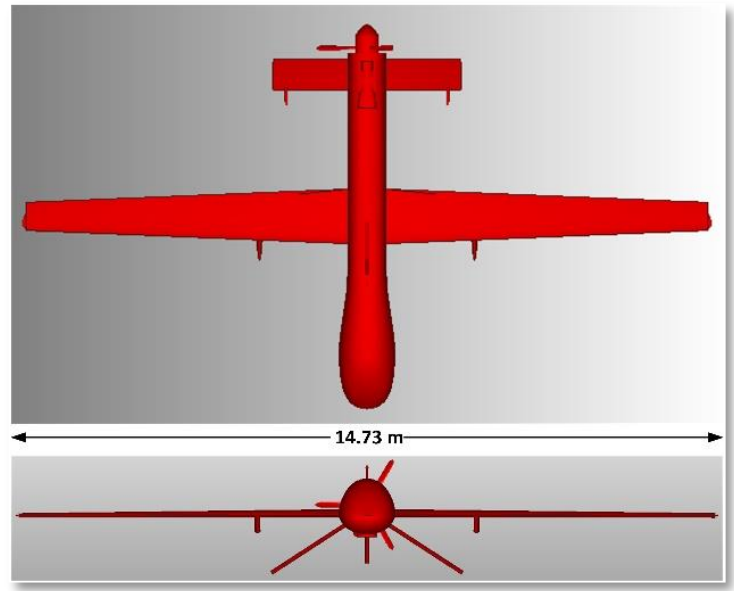

(b)

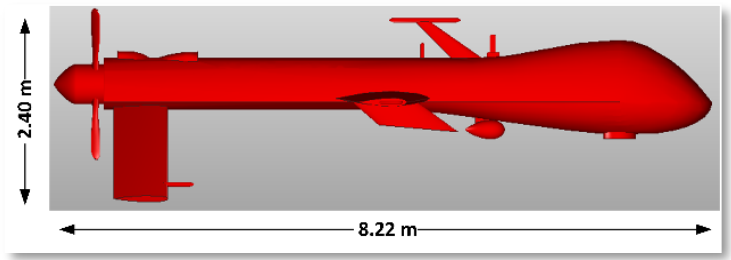

(c)

Fig. 1. Generic military UAV model: Mil-UAV (a) Perspective view, (b) Top and front view, and (c) side view

To evaluate and the RCS characteristics of Mil-UAV, two sperate RCS simulations have been carried out. Firstly, angular RCS variation for a fixed frequency has been explored. Then, the frequency RCS variation for some special look-angles that provide high scattering has also been done by the help of the RCS run. Below are the details and the outcome of these simulations. 


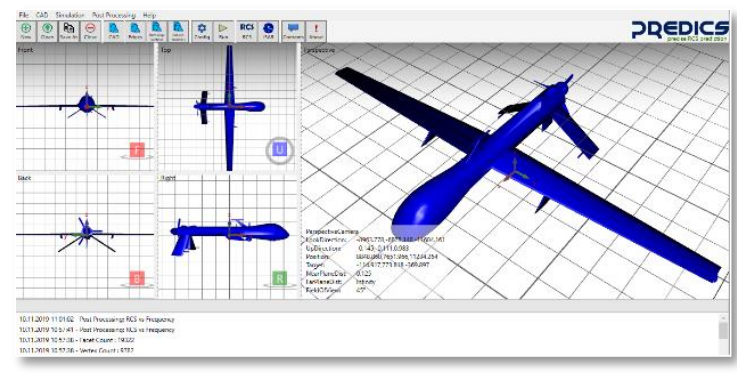

Fig. 2. PREDICS simulation screen for Mil-UAV

\subsection{RCS variation over angles}

In the first simulation, monostatic RCS variation for the azimuth and elevation look-angles at the fixed frequency of $10 \mathrm{GHz}$ has been used. The physical optics (PO) and shooting and bouncing ray (SBR) solvers of PREDICS have been utilized. The ray density merit of 10 rays per wavelength has been used for precise RCS computation. For the PREDICS simulation, horizontal angles ranging from $0^{\circ}$ (nose-on case) to $180^{\circ}$ (back-side case) for a total of 121 distinct horizontal angles and vertical angles ranging from $90^{\circ}$ (horizon case) to $111^{\circ}$ ($21^{\circ}$ below horizon case) for a total of 15 discrete vertical angles were included. These angles were decided based on the scenarios of radar looking towards an UAV in the air. Both co-polarization cases of vertical-vertical (VV) and horizontal-horizontal $(\mathrm{HH})$ were included in the RCS simulation. In Fig.3(a) and (b), RCS map results of MilUAV for VV polarization are given in polar and Cartesian coordinates, respectively.

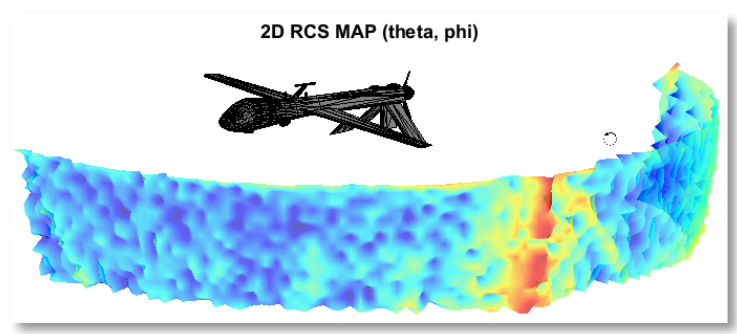

(a)

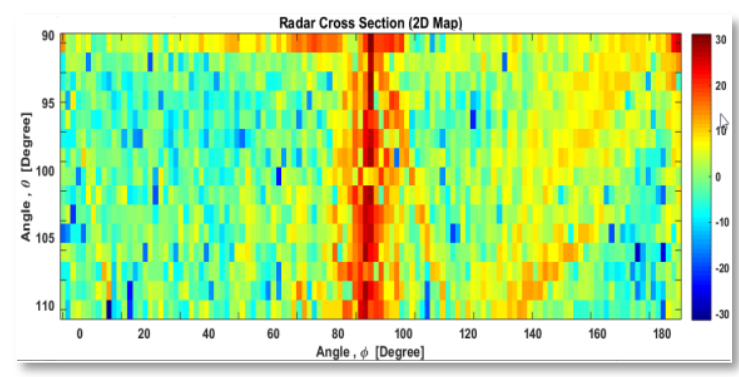

(b)

Fig. 3. 2D Angular VV-polarization RCS variation simulation result for Mil-UAV target at $10 \mathrm{GHz}$ represented using RCS map plot in (a) polar coordinates, (b) Cartesian coordinates

As it can be seen from Fig.3, the highest RCS values occur at the broad-side angles around $\phi=90^{\circ}$ for almost all vertical angles. The dynamic range of the display is selected as $65 \mathrm{~dB}$. To better interpret the highest RCS values, the one-dimensional (1D) horizontal RCS variation at $\theta=90^{\circ}$ for both $\mathrm{VV}$ and $\mathrm{HH}$ polarizations are plotted in Fig.4 as blue and red solid lines, respectively. One can fairly observe that the maximum value of RCS is equal to $31.18 \mathrm{dBsm}\left(1,312.2 \mathrm{~m}^{2}\right)$ for the look angle of $\left(\theta=90^{\circ}, \phi=90^{\circ}\right)$. If Fig. 3 is carefully analyzed, the average RCS value can be calculated as $11.64 \mathrm{dBsm}\left(10.66 \mathrm{~m}^{2}\right)$. Based on these calculated RCS results; therefore, a military UAV that has a similar size of a manned aircraft can produce RCS values that are not different than that of an aircraft. This result suggested that such UAVs can be easily detected by a radar from tens of kilometers away as it will be explored in Section 3.

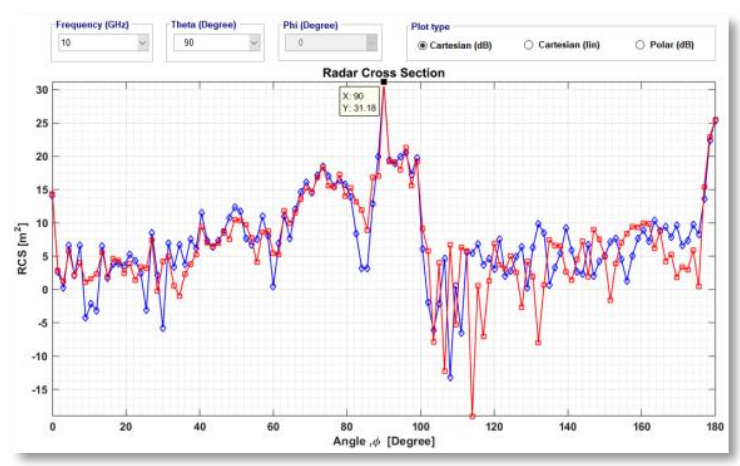

Fig. 4. 1D horizontal RCS variation simulation result for Mil-UAV at $10 \mathrm{GHz}$ and $\theta=90^{\circ}$

\subsection{RCS variation over frequencies}

For another experiment, Mil-UAV's RCS merit for various microwave frequency values has been studied. For this purpose, the monostatic RCS variation over frequencies for the fixed look angle of $\left(\theta=90^{\circ}, \phi=\right.$ $90^{\circ}$ ). Again, the ray density of 10 rays per wavelength together with PREDICS's PO+SBR solver were used. The frequency was varied from $6 \mathrm{GHz}$ to $18 \mathrm{GHz}$ for a total of 101 discrete frequencies. The aim of this simulation was to monitor if the RCS values were increasing for the higher frequencies or not. The simulation look-angles have been chosen as the lookangles that provide the highest values of RCS outcomes that were obtained in Fig. 3 and 4. In Fig. 5, the simulated RCS results for VV and $\mathrm{HH}$ polarizations are shown as blue and red solid lines, respectively. As expected, the RCS values tend to increase as the frequency increases. For the selected band of frequency values from $6 \mathrm{GHz}$ to $18 \mathrm{GHz}$, the maximum RCS value of $34.80 \mathrm{dBsm}$ $\left(3,019.95 \mathrm{~m}^{2}\right)$ was obtained for the frequency of 14.52 GHz.

\section{RCS SIMULATION OF A CIVILIAN-TYPE UAV MODEL: "CIV-UAV"}

The CAD model of a civilian type UAV model called as Civ-UAV that was used in PREDICS's RCS simulations is given in Fig.6. This model has characteristics that are common in many civilian UAVs. The size of this model is quite smaller than that of MilUAV. The perspective view of Civ-UAV is depicted Fig. 6(a). The top and front view of the model are given in Fig. 6(b). As it can be seen from the figure the wing extend of 
Civ-UAV is $240 \mathrm{~cm}$. The side view of Mil-UAV is also plotted in Fig. 6(c). The total length and height of the model are $120 \mathrm{~cm}$ and $20 \mathrm{~cm}$, respectively. During the PREDICS simulation, the material of the model is taken as PEC.

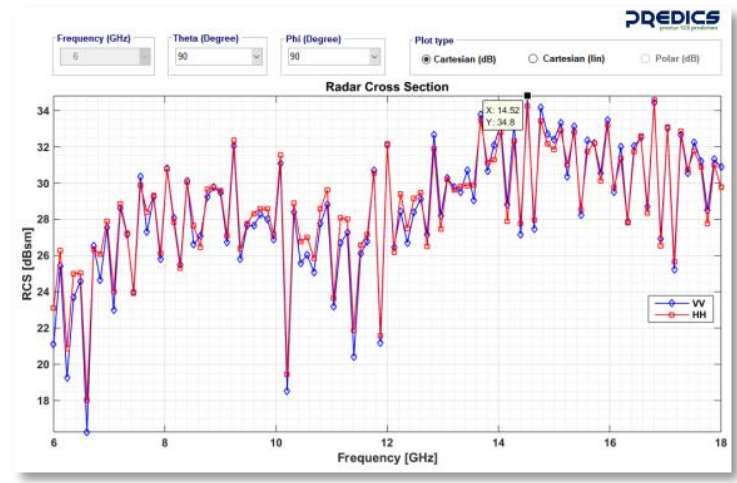

Fig. 5. Mil-UAV's RCS frequency variation for the lookangle of $\left(\theta=90^{\circ}, \phi=90^{\circ}\right)$

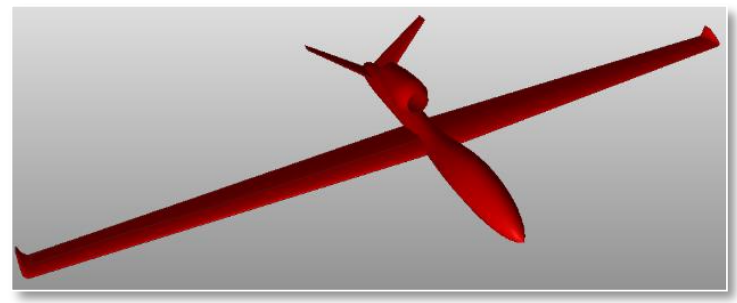

(a)

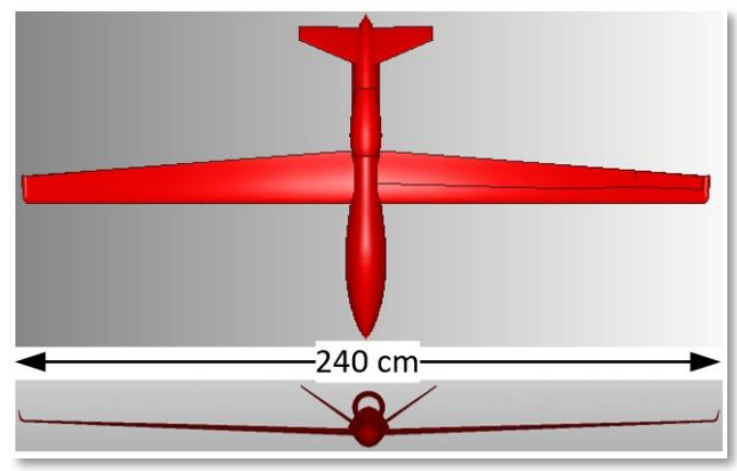

(b)

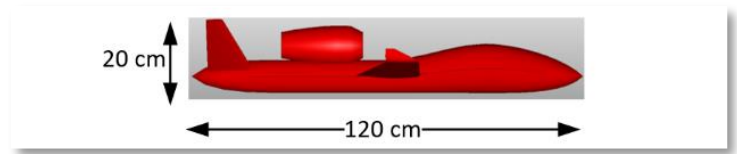

(c)

Fig. 6. Generic civilian type UAV model: Civ-UAV (a) Perspective view, (b) Top and front view, and (c) side view

PREDICS software has been used for the RCS simulation of the Civ-UAV. The RCS variation with respect to azimuth angles, elevation angles and various frequencies are given as separate studies below:

\subsection{RCS variation over angles}

To comprehend the RCS values of Civ-UAV model; first, angular RCS variation has been evaluated by collecting the VV- and HH-polarized monostatic RCS values for both the azimuth and the elevation angles at the fixed frequency of $10 \mathrm{GHz}$. Again, (PO+SBR) solver of PREDICS was used by taking 10 rays per wavelength for the ray-tracing and field-tracing procedures of the code. For the simulation, a total of 121 separate horizontal angles ranging from $0^{\circ}$ (nose-on case) to $180^{\circ}$ (back-side case) and a total of 15 different vertical angles ranging from $90^{\circ}$ (horizon case) to $111^{\circ}\left(-21^{\circ}\right.$ below horizon case) were considered similar to the simulation for Mil-UAV model. In Fig.7(a) and (b), RCS map results of Civ-UAV for VV polarization are given in polar and Cartesian coordinates, respectively. One can easily notice from this RCS map results that hot point angles are from the front (around $\phi=0^{\circ}$ ) and from the side (around $\phi=90^{\circ}$ ) from the UAV model. The simulation suggests that the RCS values are stay high around $\phi=0^{\circ}$ for almost all selected vertical angles.

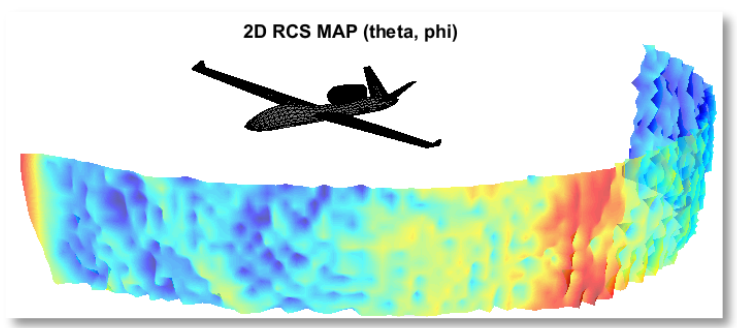

(a)

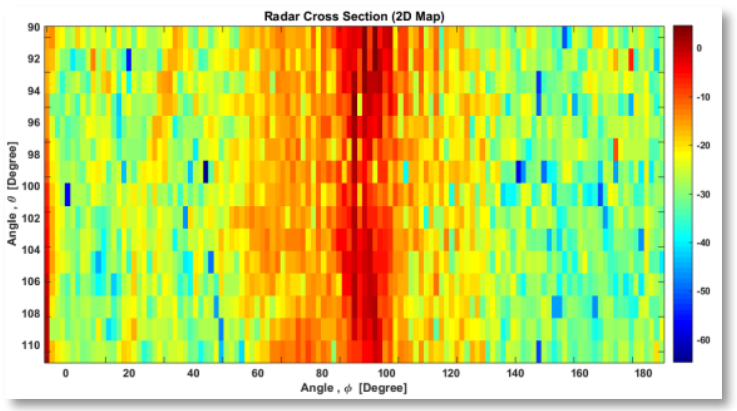

(b)

Fig. 7. 2D Angular VV-polarization RCS variation simulation result for Civ-UAV target at $10 \mathrm{GHz}$ represented using RCS map plot in (a) polar coordinates, (b) Cartesian coordinates

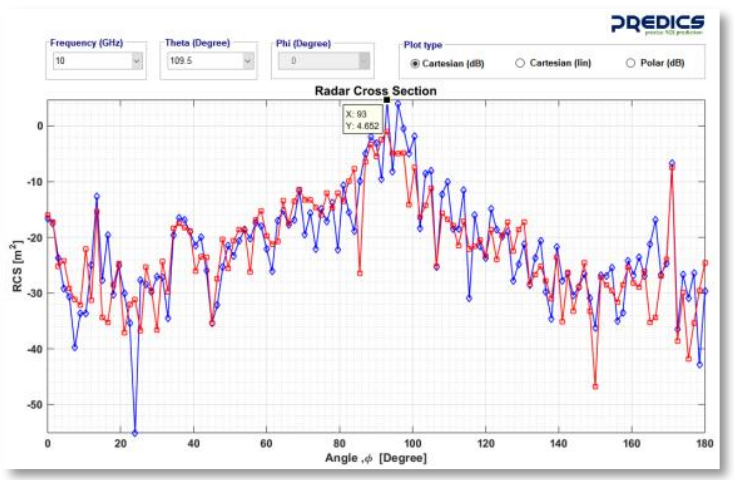

Fig. 8. Civ-UAV's RCS frequency variation for the lookangle of $\left(\theta=109.5^{\circ}, \phi=93^{\circ}\right)$ 
To better comprehend the uppermost RCS values, the 1D horizontal RCS variation at $\theta=109.5^{\circ}$ for both VV and $\mathrm{HH}$ polarizations are given in Fig. 8 as blue and red solid lines, respectively. It can be easily read from the figure that the highest RCS value is equal to $4.652 \mathrm{dBsm}$ $\left(2.92 \mathrm{~m}^{2}\right)$ for the look angle of $\left(\theta=109.5^{\circ}, \phi=93^{\circ}\right)$. Then, the average RCS value of data in Fig. 7 has been calculated as $-12.69 \mathrm{dBsm}\left(0.054 \mathrm{~m}^{2}\right)$.

\subsection{RCS variation over frequencies}

In the second RCS study for the Civ-UAV model, monostatic RCS variation over frequencies ranging from $6 \mathrm{GHz}$ to $18 \mathrm{GHz}$ for a total of 100 discrete frequencies at the look angle of $\theta=109.5^{\circ}$ and $\phi=93^{\circ}$ has been examined. The resultant PREDICS's RCS simulation result for VV- and HH-polarizations are given in Fig. 9 as blue and red solid lines, respectively. It is observed that RCS values are somewhat fluctuating between $-25 \mathrm{dBsm}$ to $5 \mathrm{dBsm}$ for the selected frequencies of interest. The maximum monostatic RCS value of $4.96 \mathrm{dBsm}\left(3.13 \mathrm{~m}^{2}\right)$ occurs for $\mathrm{HH}$ polarization at $14.36 \mathrm{GHz}$.

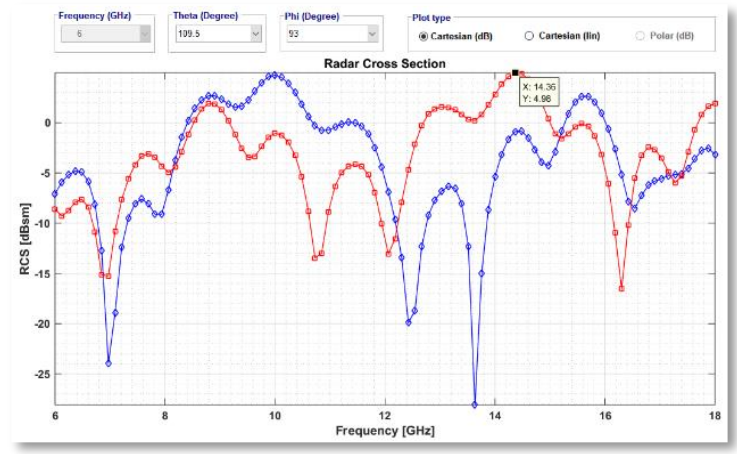

Fig. 9. Civ-UAV's RCS frequency alteration for the lookangle of $\left(\theta=109.5^{\circ}, \phi=93^{\circ}\right)$

\section{RADAR DETECTABILITY STUDY FOR THE UAV MODELS}

After completing RCS simulations that provided the RCS analysis studies for the selected UAV models, a radar detectability study has also been carried out to assess the detection ranges of both UAV models.

\subsection{Range of radar detection}

The detection range of radar; i.e., the far most distance of the target that can be spotted over the noise floor by the radar can be calculated by using the following formula (Özdemir, 2012):

$$
R_{\text {det }}=\left(\frac{P_{\text {rad }}}{P_{\min }} \cdot \frac{(\lambda G)^{2}}{(4 \pi)^{3}} \cdot \sigma\right)^{1 / 4}
$$

Here, $P_{\text {rad }}$ represents the radiated power from the radar and $P_{\min }$ is the sensitivity of the radar or the minimum power level that the radar receiver can sense. In the above equation, $\lambda$ is the wavelength that corresponds to the operation frequency of the radar, $G$ is the gain of the radar antenna and $\sigma$ is the RCS value of the target that is tried to be detected.

\subsection{Detectability study of Mil-UAV model}

The detection range of Mil-UAV model has been investigated by putting nominal values for the variables within the formula in Eqn. (1). This study is explored via the Table 1 by using the RCS results that was obtained by PREDICS. The following radar parameters were assumed for a highly sensitive detection radar: Two different values of radiated powers levels of $20 \mathrm{~dB}(100 \mathrm{~W})$ and 24 $\mathrm{dB}(251 \mathrm{~W})$ were considered. The radar was also assumed to be working in two different sensitivity levels of $-95 \mathrm{~dB}$ and $-117 \mathrm{~dB}$. The gain of the radar antenna is taken as 44 $\mathrm{dBi}$ that is a nominal figure for a directive radar antenna.

The corresponding detection ranges for Mil-UAV model are listed in the last column of Table 1. Based on the assumed parameters and the predicted RCS values of the UAV target at $10 \mathrm{GHz}$, it can be observed from the Table 1 that the minimum detectable range is calculated as about $5.7 \mathrm{~km}$ for the average RCS value of $11.64 \mathrm{dBsm}$ that represents the worst-case scenario. On the other hand, the best case happens when the maximum value of RCS, $31.18 \mathrm{dBsm}$ is used for the parameters given in the fourth row of Table 1. For this situation, the minimum detectable range can extend up to $83 \mathrm{~km}$. In summary, the detection of a UAV that has dimensions similar to Mil-UAV can be possible at the ranges from 5 to $80 \mathrm{~km}$.

Table 1. Detection range of Mil-UAV model at $f=10 \mathrm{GHz}$

\begin{tabular}{ccccc}
\hline $\boldsymbol{\sigma}(\mathbf{d B s m})$ & $\boldsymbol{P}_{\boldsymbol{r a d}}(\mathbf{d B})$ & $\boldsymbol{P}_{\boldsymbol{m i n}}(\mathbf{d B})$ & $\boldsymbol{G}(\mathbf{d B i})$ & $\boldsymbol{R}_{\boldsymbol{d e t}}(\boldsymbol{m})$ \\
\hline 31.18 & 20 & -95 & 44 & $18,558 \mathrm{~m}$ \\
31.18 & 20 & -117 & 44 & $65,848 \mathrm{~m}$ \\
31.18 & 24 & -95 & 44 & $23,364 \mathrm{~m}$ \\
31.18 & 24 & -117 & 44 & $\mathbf{8 2 , 8 9 8} \mathbf{~}$ \\
11.64 & 20 & -95 & 44 & $\mathbf{5 , 6 9 8} \mathbf{~ m}$ \\
11.64 & 20 & -117 & 44 & $20,218 \mathrm{~m}$ \\
11.64 & 24 & -95 & 44 & $7,173 \mathrm{~m}$ \\
11.64 & 24 & -117 & 44 & $25,452 \mathrm{~m}$ \\
\hline
\end{tabular}

\subsection{Detectability study of Civ-UAV model}

A similar detectability study has been done for the Civ-UAV model that is much smaller than the its military counterpart. The same radar parameters were used as utilized in the previous study. For the calculation of the minimum detection range of Civ-UAV model, maximum RCS value of $4.652 \mathrm{dBsm}$ and average RCS value of $12.69 \mathrm{dBsm}$ were used in calculating the minimum detection ranges that are listed along the last column of Table 2. For the different radar parameters that are given in other columns of Table 2, the minimum detection range is varying between approximately $1.5 \mathrm{~km}$ and $18 \mathrm{~km}$ as observed from the last column of the table. Considering the UAVs' nominal cruising speed values ranging from 50 to 100 knots, range values around a few kilometers may not be adequate for some scenarios. 
Table 2. Detection range of Civ-UAV model at $f=10 \mathrm{GHz}$

\begin{tabular}{ccccc}
\hline $\boldsymbol{\sigma}(\mathbf{d B s m})$ & $\boldsymbol{P}_{\boldsymbol{r a d}}(\mathbf{d B})$ & $\boldsymbol{P}_{\boldsymbol{m i n}}(\mathbf{d B})$ & $\boldsymbol{G}(\mathbf{d B i})$ & $\boldsymbol{R}_{\text {det }}(\boldsymbol{m})$ \\
\hline 4.652 & 20 & -95 & 44 & $4,031 \mathrm{~m}$ \\
4.652 & 20 & -117 & 44 & $14,303 \mathrm{~m}$ \\
4.652 & 24 & -95 & 44 & $5,0751 \mathrm{~m}$ \\
4.652 & 24 & -117 & 44 & $\mathbf{1 8 , 0 0 7} \mathbf{~}$ \\
-12.69 & 20 & -95 & 44 & $\mathbf{1 , 4 8 5} \mathbf{~ m}$ \\
-12.69 & 20 & -117 & 44 & $5,270 \mathrm{~m}$ \\
-12.69 & 24 & -95 & 44 & $1,870 \mathrm{~m}$ \\
-12.69 & 24 & -117 & 44 & $6,635 \mathrm{~m}$ \\
\hline
\end{tabular}

\section{CONCLUSION}

In this paper, we have represented a quantitative analysis of RCS from two different UAV models; namely the Mil-UAV and Civ-UAV to assess their visibility characteristics by a nominal radar. We have utilized the high-frequency RCS simulator tool of PREDICS to perform the required electromagnetic simulations to predict their RCS values at X-band. By the help of PREDICS software, the monostatic RCS simulations of the model UAVs based on various angles and frequencies have been accomplished.

It was obtained from the simulations that RCS values for the large-size model of Mil-UAV vary vastly such as $11 \mathrm{dBsm}$ on average and $31 \mathrm{dBsm}$ at maximum at $10 \mathrm{GHz}$. The radar detectability study for this model has provided minimum detection ranges on the order to tens of kilometers. Such values of detection figures are good enough to sense and take measures for hostile usages of similar UAVs. On the other hand, a civilian model of CivUAV has yielded RCS values such as $-12.67 \mathrm{dBsm}$ on average and $4.652 \mathrm{dBsm}$ at maximum. After completing a similar study as in the case of a Mil-UAV model, it is calculated that the minimum radar detection range extends from $1.5 \mathrm{~km}$ to a few kilometers. These range values may not be good enough for a radar to detect and take necessary measures to secure the targeted area of a hostile UAV.

\section{ACKNOWLEDGEMENTS}

This work was supported by Mersin University Scientific Research Unit under Project No. 2015-TP31160.

\section{REFERENCES}

Akar, A. (2017). Evaluation Of Accuracy of Dems Obtained From UAV-Point Clouds for Different Topographical Areas. International Journal of
Engineering and Geosciences, 2 (3), 110-117.

Ananenkov, A. E., Marin, D. V., Nuzhdin, V. M., Rastorguev V. V., and Sokolov, P. V. (2018) "Possibilities to Observe Small-Size UAVs in the Prospective Airfield Radar," 2018 20th International Conference on Transparent Optical Networks (ICTON), Bucharest, 2018, pp. 1-6.

Özdemir C., Inverse Synthetic Aperture Radar Imaging with MATLAB Algorithms (2012), John Wiley \& Sons, March 2012, Hoboken, New Jersey, ISBN: 978-0-47028484-1.

Özdemir, C., Yılmaz, B., and Kırık, Ö. (2014a), "PREDICS: A new GO-PO based ray launching simulator for the calculation of electromagnetic scattering and RCS from electrically large and complex structures," Turkish Journal of Electrical Engineering \& Computer Sciences, Vol. 22, 1255 - 1269

Özdemir, C., Y1lmaz, B., Kırık, Ö., Sütcüoğlu, Ö. (2014b), "A Fast and Efficient RCS Calculation and ISAR Image Formation Tool: PREDICS", 10th European Conference on Synthetic Aperture Radar (EUSAR 2014), Berlin.

Pieraccini, M., Miccinesi, L. and Rojhani, N. (2017) "RCS measurements and ISAR images of small UAVs," in IEEE Aerospace and Electronic Systems Magazine, vol. 32, no. 9, pp. 28-32, September 2017.

Ryapolov, I., Sukharevsky O., and Vasilets, V. (2014) "Radar cross-section calculation for unmanned aerial vehicle," 2014 International Conference on Mathematical Methods in Electromagnetic Theory, Dnipropetrovsk, pp. 258-261.

Ulvi, A. Toprak, A. (2016). "Investigation Of ThreeDimensional Modelling Availability Taken Photograph Of The Unmanned Aerial Vehicle; Sample Of Kanlidivane Church.”, International Journal of Engineering and Geosciences, 1 (1), pp. 1-7.

Ulvi, A. (2018). "Analysis of The Utility of the Unmanned Aerial Vehicle (UAV) in Volume Calculation by Using Photogrammetric Techniques." International Journal of Engineering and Geosciences, 3 (2), pp. 43-49. 